\title{
Prime Distribution in Pythagorean Triples (1)
}

\section{Chun-Xuan Jiang*}

Institute for Basic Research, Palm Harbor, P.O. Box 3924, Beijing 100854, P.R. China

\section{Mini-Review}

Using Jiang function we study the prime distribution in Pythagorean triples.

\section{Pythagorean triples}

$a^{2}+b^{2}=c^{2}$,

In comprime integers must be of the form:

$a=x^{2}-y^{2}, \quad b=2 x y, \quad c=x^{2}+y^{2}$,

Where $x$ and $y$ are coprime integers.

Theorem 1: From eqn. (2) we have,

$a=(x+y)(x-y)$

Let $x-y=1$ and $a=x+y=P_{1}$, we have,

$P_{1}^{2}=(x+y)^{2}=x^{2}+y^{2}+2 x y=c+b$,

$1=(x-y)^{2}=x^{2}+y^{2}-2 x y=c-b$

From eqns. (4) and (5) we have,

$a=P_{1}, \quad b=\frac{P_{1}^{2}-1}{2}, \quad c=\frac{P_{1}^{2}+1}{2}=P_{2}$

There are infinitely many primes $P_{1}$ such that $P_{2}$ is a prime.

Proof: We have Jiang function [1]

$J_{2}(\omega)=\prod_{P>2}(P-1-\chi(P))$,

where $\omega=\prod_{P \geq 2} P, \chi(P)$ is the number of solutions of congruence

$q^{2}+1 \equiv 0(\bmod P), q=1, \cdots, P-1$.

From (8) we have,

$\chi(P)=1+(-1)^{\frac{P-1}{2}}$

Substituting (9) into (7) we have

$J_{2}(\omega)=\prod_{P>2}\left(P-2-(-1)^{\frac{P-1}{2}}\right) \neq 0$

Since $J_{2}(\omega) \neq 0$, we prove that there are infinitely many prime $P_{1}$, such that $P_{2}$ is a prime.

We have the best asymptotic formula [1]

$\pi_{2}(N, 2)=\mid\left\{P_{1} \leq N: P_{2}=\right.$ prime $\} \mid \sim \frac{J_{2}(\omega) \omega}{2 \phi^{2}(\omega)} \frac{N}{\log ^{2} N}=\left(1-\frac{1+P(-1)^{\frac{P-1}{2}}}{(P-1)^{2}}\right) \frac{N}{\log ^{2} N}$

where $\phi(\omega)=\prod_{P \geq 2}(P-1)$.

Theorem 2: Let $x+y=P_{1}$ and $x-y=P_{1}-2$, we have $a=P_{1}\left(P_{1}-2\right)$ and,

$$
\begin{aligned}
& P_{1}^{2}=(x+y)^{2}=c+b, \\
& \left(P_{1}-2\right)^{2}=(x-y)^{2}=c-b
\end{aligned}
$$

From eqns. (12) and (13) we have,

$a=P_{1}\left(P_{1}-2\right), b=\frac{P_{1}^{2}-\left(P_{1}-2\right)^{2}}{2}, c=\frac{P_{1}^{2}+\left(P_{1}-2\right)^{2}}{2}=P_{2}$

There are infinitely many primes $P_{1}$ such that $P_{2}$ is a prime

Proof: We have Jiang function [1]

$J_{2}(\omega)=\prod_{P>2}(P-1-\chi(P))$,

Where $\chi(P)$ is the number of solutions of congruence

$q^{2}+(q-2)^{2} \equiv 0(\bmod P), q=1, \cdots, P-1$.

From (16) we have,

$\chi(P)=1+(-1)^{\frac{P-1}{2}}$

Substituting (17) into (15) we have,

$J_{2}(\omega)=\prod_{P>2}\left(P-2-(-1)^{\frac{P-1}{2}}\right) \neq 0$

Since $J_{2}(\omega) \neq 0$, we prove that there are infinitely many prime $P_{1}$ such that $P_{2}$ is a prime.

We have the best asymptotic formula [1]

$\pi_{2}(N, 2)=\mid\left\{P_{1} \leq N: P_{2}=\right.$ prime $\} \mid \sim\left(1-\frac{1+P(-1)^{\frac{P-1}{2}}}{(P-1)^{2}}\right) \frac{N}{\log ^{2} N}$

Theorem 3: Let $x-y=1$ and $a=x+y=P_{1}^{2}$, we have,

$a=P_{1}^{2}, b=\frac{P_{1}^{4}-1}{2}, c=\frac{P_{1}^{4}+1}{2}=P_{2}$

There are infinitely many primes $P_{1}$ such that $P_{2}$ is a prime.

Proof: We have Jiang function [1],

$J_{2}(\omega)=\prod_{P>2}(P-1-\chi(P))$,

Where $\chi(P)$ is the number of solutions of congruence,

$q^{4}+1 \equiv 0(\bmod P), q=1, \cdots, P-1$.

From (22) we have,

$\chi(P)=4$ if $8 \mid P-1, \quad \chi(P)=0$ otherwise

*Corresponding author: Chun-Xuan Jiang, Institute for Basic Research, Palm Harbor, P.O. Box 3924, Beijing 100854, P.R. China, Tel: +1-727-688 3992; E-mail: jcxuan@sina.com

Received March 10, 2017; Accepted July 29, 2017; Published July 31, 2017

Citation: Jiang CX (2017) Prime Distribution in Pythagorean Triples (1). J Generalized Lie Theory Appl 11: 276. doi: 10.4172/1736-4337.1000276

Copyright: ( 2017 Jiang CX. This is an open-access article distributed under the terms of the Creative Commons Attribution License, which permits unrestricted use, distribution, and reproduction in any medium, provided the original author and source are credited. 
Page 2 of 2

Since $J_{2}(\omega) \neq 0$, we prove that there are infinitely many prime $P_{1}$ such that $P_{2}$ is a prime.

We have the best asymptotic formula [1]:

$\pi_{2}(N, 2)=\mid\left\{P_{1} \leq N: P_{2}=\right.$ prime $\} \mid \sim \frac{J_{2}(\omega) \omega}{4 \phi^{2}(\omega)} \frac{N}{\log ^{2} N}$,
These results are in wide use in biological, physical and chemical fields.

\section{References}

1. Chun-Xuan Jiang, Jiang function $J_{n+1}(\omega)$ in prime distribution. 